\title{
The minimum number of multiplicity 1 eigenvalues among real symmetric matrices whose graph is a nonlinear tree
}

\author{
https://doi.org/10.1515/spma-2021-0158
}

Received July 29, 2021; accepted January 7, 2022

\begin{abstract}
In the study of eigenvalues, multiplicities, and graphs, the minimum number of multiplicities equal to 1 in a real symmetric matrix with graph $G, U(G)$, is an important constraint on the possible multiplicity lists among matrices in $\mathcal{S}(G)$. Of course, the structure of $G$ must determine $U(G)$, but, even for trees, this linkage has proven elusive. If $T$ is a tree, $U(T)$ is at least 2, but may be much greater. For linear trees, recent work has improved our understanding. Here, we consider nonlinear trees, segregated by diameter. This leads to a new combinatorial construct called a core, for which we are able to calculate $U(T)$. We suspect this bounds $U(T)$ for all nonlinear trees with the given core. In the process, we develop considerable combinatorial information about cores.
\end{abstract}

Keywords: Eigenvalue; Graph of a matrix; Multiplicity list; Nonlinear tree; $U(T)$

MSC: Primary: 15A18, 05C50 ; Secondary: 15B57

\section{Introduction}

The graph of an $n$-by- $n$ real symmetric matrix $A=\left(a_{i j}\right)$ is an undirected, simple graph $G(A)$ on vertices $\{1,2, \cdots, n\}$ in which there is an edge $\{i, j\}$ if and only if $a_{i j} \neq 0$. Given a graph $G$, we consider the set $\mathcal{S}(G)$ of all real symmetric $A$ such that $G(A)=G$. Each element of $\mathcal{S}(G)$ enjoys a list of ordered multiplicities for its eigenvalues, i.e., an ordered partition of $n$, in which the order corresponds to the numerical order of the underlying, necessarily real, eigenvalues. It is known [10] that if $G=T$ is a tree, then the first and last multiplicity in every ordered list are both 1 . We also consider "unordered" multiplicity lists, in which the elements of the partition are simply listed in descending order. For a given graph $G$, the catalog of all such unordered lists that occur among matrices in $\mathcal{S}(G)$ is denoted $\mathcal{L}(G)$, while the ordered catalog is denoted $\mathcal{L}_{o}(G)$. Of course, determining the catalog, especially for trees, has been the subject of much prior research. The recent book [10] is a good general reference, and it provides a database for $\mathcal{L}(T)$ for all trees on fewer than 13 vertices.

An important constraint on the catalogs for trees is the minimum number of 1's occurring among the lists for a tree $T$, which we denote as $U(T)$. For any tree, $U(T) \geqslant 2$, and it may well be larger. A high degree vertex (HDV) of a tree is simply one of degree at least 3. A tree is called linear if all its HDV's lie on a single induced path of the tree; otherwise it is called "nonlinear" (NL). Any tree with fewer than 4 HDV's is linear, and the first NL tree has 10 vertices (see display in Figure 1).

^Corresponding Author: Wenxuan Ding: Department of Mathematics, College of William \& Mary, E-mail: wding@email.wm.edu

Charles R. Johnson: Department of Mathematics, College of William \& Mary, E-mail: crjohn@wm.edu 
There is remarkable information about the catalog of linear trees based upon the so-called linear superposition principle (LSP) [8, 13]. In view of recent work on $U(T)$ for linear trees[2, 3], our interest here is in better understanding $U(T)$ for NL trees.

Of course, it is natural to relate important aspects of multiplicity lists to the structure of a tree. Examples include the following. The path cover number, $P(T)$, is the fewest vertex disjoint induced paths of the tree that cover all its vertices. For the 10-vertex NL tree, $P(T)=4$. It is known that the maximum multiplicity, $M(T)$, appearing in the catalog $\mathcal{L}(T)$, for a tree $T$, is exactly $P(T)$ [4]. Also, the diameter, $d(T)$, of a tree $T$, is simply the greatest number of vertices in an induced path of $T$, and the minimum number of distinct eigenvalues (the number of parts in a shortest partition) of a matrix in $\delta(T)$ is at least $d(T)[5,9]$. We also use diameter to refer to a path in $T$ with length $d(T)$. Moreover, $U(T) \geqslant 2 d(T)-n$, in which $n$ is the number of vertices of $T$ [1].

Because there is no known analog of the LSP for NL trees, it is more difficult to understand $U(T)$ for NL trees. So, for NL trees, we develop some new combinatorial ideas, primarily the "core", and relate them to tree structure and to $U(T)$. In the process, we are able to enumerate some tree parameters in terms of cores with a given diameter. In the case of linear trees, prior work has given some detailed information about $U(T)$, mostly using the LSP [1-3]. This includes explicit formulae in the case of 1 or 2 HDV's and results about the possible changes in $U(T)$ with the addition of a vertex. (In particular, vertex addition is done via pendent vertex addition, in which a new edge and a vertex pendent at an existing vertex are added, or edge subdivision, in which a new vertex of degree 2 is positioned along an existing edge.) If a corresponding incremental result is true in the NL case, a conjecture we make based on new results here would give strong information about $U(T)$ for NL trees.

\section{Background}

Throughout, we employ standard submatrix notation. Let $A$ be an $n$-by- $n$ matrix. If $\alpha \subset\{1, \ldots, n\}$ is an index set, then $A[\alpha]$ is the principal submatrix of $A$ in the rows and columns indexed by $\alpha$, and $A(\alpha):=$ $A[\{1, \ldots, n\} \backslash \alpha]$. In the case when $\alpha=\{v\}$, we abbreviate $A(\{v\})$ to $A(v)$. Observe that the graph $T[\alpha]$ is the subgraph of $T$ induced by the vertices corresponding to $\alpha$. If $A \in \mathcal{S}(T)$, then $A[\alpha] \in \mathcal{S}(T[\alpha])$, and we often think of the matrix and graph interchangeably. We write $m_{A}(\lambda)$ for the multiplicity of the eigenvalue $\lambda$ in the matrix $A$ (subscript $A$ sometimes omitted). A classical and fundamental theorem for this study is the interlacing inequalities for Hermitian (real symmetric) matrices. An immediate consequence of this theorem is that for any eigenvalue $\lambda$ and $i \in\{1, \ldots, n\},\left|m_{A}(\lambda)-m_{A(i)}(\lambda)\right| \leq 1$. That is, the multiplicity of an eigenvalue of a real symmetric matrix changes by at most 1 if a principal submatrix of size one smaller is extracted.

Let $A$ be a real symmetric matrix whose graph is a tree $T$. The removal of a vertex $v$ from a tree $T$ corresponds to the removal of a row and column with the same index $v$ from $A$. When $v$ is deleted from $T$, a forest of several components $T_{1}, \ldots, T_{\operatorname{deg}_{v}}$ remains. The corresponding matrix is $A(v)=A\left[T_{1}\right] \oplus \cdots \oplus A\left[T_{\operatorname{deg}_{v}}\right]$. The Parter-Wiener, etc. theorem is one of the most important tools in the study of eigenvalues, multiplicities, and graphs. The most general form of the theorem is given in [7].

Theorem 2.1. Let $T$ be a tree and $A$ a matrix in $\mathcal{S}(T)$. Let $\sigma(A)$ denote the spectrum of A. Suppose that there is a vertex $v$ of $T$ and a real number $\lambda$ such that $\lambda \in \sigma(A) \cap \sigma(A(v))$. Then

(1) there is a vertex $u$ of $T$ such that $m_{A(u)}(\lambda)=m_{A}(\lambda)+1$;

(2) if $m_{A}(\lambda) \geq 2$, then $\lambda \in \sigma(A) \cap \sigma(A(v))$ is automatically satisfied and $u$ may be chosen so that $\operatorname{deg}_{T}(u) \geq 3$ and so that there are at least three components $T_{1}, T_{2}$, and $T_{3}$ of $T \backslash u$ such that $m_{A\left[T_{i}\right]}(\lambda) \geq 1, i=1,2,3$; and

(3) if $m_{A}(\lambda) \geq 1$, then $u$ may be chosen so that $\operatorname{deg}_{T}(u) \geq 2$ and so that there are two components $T_{1}$ and $T_{2}$ of $T \backslash u$ such that $m_{A\left[T_{i}\right]}(\lambda) \geq 1, i=1,2$. 
We call a vertex $v$ meeting the requirement in the above theorem a Parter vertex of $T$ for $\lambda$ in $A$ (i.e., $v$ is Parter for $\lambda$ in $A$.) In other words, $v$ is Parter for $\lambda$ if $m_{A(v)}(\lambda)=m_{A}(\lambda)+1$ and $\lambda$ is an eigenvalue of the submatrices corresponding to at least two of the connected components of $T-v$.

Some classes of trees have been studied extensively. A (simple) star is a tree on $n$ vertices having a vertex of degree $n-1$. This vertex is called the center of the star. A generalized star ( $g$-star) is a tree with at most one HDV; moreover, the HDV (or a degree 2 vertex if there is no HDV) is called the central vertex of the g-star. A $\mathrm{g}$-star consists of several paths ( $\mathrm{arms}$ ) hanging from the central vertex. For both stars and g-stars, the choice of Parter vertex for any multiple eigenvalue is obvious as there is no other HDV in them.

Lastly, we introduce a construction technique for multiplicity lists with multiple eigenvalues: the method of eigenvalue assignments (to subtrees for which possible spectra are known). This technique is an informal method of constructing multiplicity lists using Parter vertices and several coincidences of eigenvalues among various subtrees. A realization of an assignment verifies the existence of a desired multiplicity list. Definition 2.2 states the formalization of eigenvalue assignment. More details can be found in [10].

Definition 2.2. Let $T$ be a tree on $n$ vertices and let

$$
\left(p_{1}, p_{2}, \ldots, p_{k}, 1^{n-\sum_{i=1}^{k} p_{i}}\right)
$$

be a non-increasing list of positive integers, with $\sum_{i=1}^{k} p_{i} \leqslant n$. The notation $1^{l}$ denotes that the last $l$ entries of the list are 1 . These will be the desired eigenvalue multiplicities. Note that some of the $p_{i}$ 's may be 1 . Then, an assignment $\mathcal{A}$ is a collection $\mathcal{A}=\left\{\mathcal{A}_{1}, \ldots, \mathcal{A}_{k}\right\}$ of $k$ collections $\mathcal{A}_{i}$ of subtrees of $T$, corresponding to eigenvalues with multiplicities $p_{i}$, with the following properties.

1. (Specification of Parter vertices) For each $i$, there exists a set $V_{i}$ of vertices of $T$ such that

(1a) Each subtree in $\mathcal{A}_{i}$ is a connected component of $T-V_{i}$.

(1b) $\left|\mathcal{A}_{i}\right|=p_{i}+\left|V_{i}\right|$.

(1c) For each vertex $v \in V_{i}$, there exists a vertex $x$ adjacent to $v$ such that $x$ is in one of the subtrees in $\mathcal{A}_{i}$.

2. (No overloading) We require that no subtree $S$ of $T$ is assigned more than $|S|$ eigenvalues; define $c_{i}(S)=$ $\left|\mathcal{A}_{i} \cap \mathcal{Z}(S)\right|-\left|V_{i} \cap S\right|$, the difference between the number of subtrees contained in $S$ and the number of Parter vertices in $S$ for the $i^{\text {th }}$ multiplicity. Then we require that $\sum_{i=1}^{k} \max \left(0, c_{i}(S)\right) \leq|S|$ for each $S \in Z(T)$. If this condition is violated at any subtree, then that subtree is said to be overloaded. Notation: if $V$ is a set of vertices and $G$ is a graph, then $V \cap G$ denotes the set of vertices in both $V$ and $G$. And $Z(T)$ denotes the collection of all subtrees of $T$, including $T$.

Theorem 2.3. If $T$ is a tree and $q \in \mathcal{L}(T)$ includes multiplicities greater than 1, then there is an assignment for $q$.

We use an example from [10] to illustrate this technique.

Example 2.4. Consider the following tree $T$.

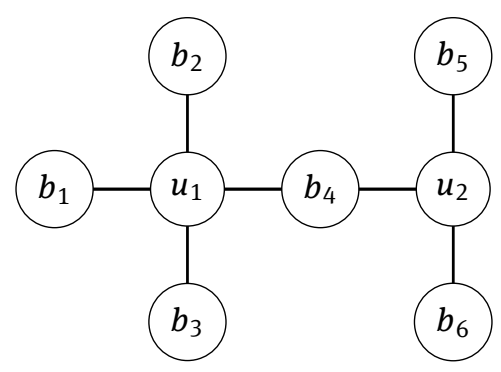

The multiplicity list $\left(3,2,1^{3}\right) \in \mathcal{L}(T)$ because of the realizable assignment in which $u_{1}$ is Parter for $\alpha$ such that $m(\alpha)=3$ and $u_{2}$ is Parter for $\beta$ such that $m(\beta)=2$. To be specific, $\alpha$ appears four times in $T-u_{1}$ : on 
the three neighbors of $u_{1}$ and once on the subtree to the right; $\beta$ appears three times in $T-u_{2}$ : on the two neighbors of $u_{2}$ and once on the subtree to the left. Formally, the assignment is summarized as follows.

$\mathcal{A}_{1}=\left\{\left\{b_{1}\right\},\left\{b_{2}\right\},\left\{b_{3}\right\},\left\{b_{4}, b_{5}, b_{6}, u_{2}\right\}\right\}$ with $V_{1}=\left\{u_{1}\right\}$ for $\alpha$.

$\mathcal{A}_{2}=\left\{\left\{b_{5}\right\},\left\{b_{6}\right\},\left\{u_{1}, b_{1}, b_{2}, b_{3}, b_{4}\right\}\right\}$ with $V_{2}=\left\{u_{2}\right\}$ for $\beta$.

Nevertheless, there is no eigenvalue assignment for the multiplicity list $\left(2,2,2,1^{2}\right)$, hence $\left(2,2,2,1^{2}\right) \notin$ $\mathcal{L}(T)$. In particular, one of the two HDV's $\left(u_{1}\right.$ and $u_{2}$ ) would have to be Parter for two of the multiplicity 2 eigenvalues. However, neither has enough branches of sufficient size to assign the two eigenvalues a total of six times. Furthermore, not $\left(5,1^{3}\right)$ but $\left(4,1^{4}\right)$ is possible by letting both $u_{1}$ and $u_{2}$ be Parter for the same eigenvalue. Also, notice that since $\left(3,2,1^{3}\right) \in \mathcal{L}(T), U(T) \leqslant 3$. In fact, according to the database of all trees on fewer than 13 vertices [10], $U(T)=3$.

\section{Cores}

\subsection{The Notion of Core}

We propose a way to classify NL trees by diameter.

Definition 3.1. An NL core of diameter $d(\geqslant 5)$ is a NL tree, minimal with respect to the number of vertices. Diameter $d$ cores are not unique $(d \geqslant 6)$, but every diameter $d$ NL tree is the result of pendent vertex addition to one or more diameter $d$ cores.

Lemma 3.2. Any diameter d core contains only 4 HDV's.

Proof. Suppose the core $C$ had five (or more) HDV's. Choose one, say $v$, so that the remaining HDV's also do not lie on a single path. A diameter, the longest induced path, can go through at most two neighbors of $v$. Remove all other neighbors of $v$ in $C$, making $v$ no longer an HDV, and giving a new tree $C^{\prime} . C^{\prime}$ still has diameter $d$ and is still NL. So, $C$ was not a core.

Now, we give a characterization of diameter $d$ cores.

Proposition 3.3. An NL tree $T$ with diameter $d$ is a diameter $d$ core if and only if the number of vertices in $T$ is $d+5$.

Proof. First, adding five vertices to a path on $d$ vertices can result in an NL tree with diameter $d$. In particular, we choose three interior vertices on the path, add a pendent vertex to the first and third vertex, and attach a 3-path to the second vertex (with the middle vertex of the 3-path adjacent to it.) Since a core, say $T$, has the fewest vertices, $n(T) \leqslant d+5$.

Second, $n(T) \geqslant d+5$. By Lemma 3.2, among the four HDV's in $T$, the diameter goes through at most three of them. Then, at least five vertices are not on the diameter - the last HDV that is not on the diameter, say $v$, at least two of $v$ 's neighbors, and one neighbor for each of at least two HDV's that are on the diameter. So, $n(T) \geqslant d+5$. In conclusion, $n(T)=d+5$.

Remark 3.4. We classify NL trees by diameter because cores group NL trees in a systematic way. We remark the following.

1. (Finiteness) For each $d$, there are finitely many diameter $d$ cores, up to isomorphism.

2. (Infinity) Each diameter $d$ core generates an infinite family of diameter $d$ NL trees via a sequence of pendent additions of vertices without increasing the diameter.

3. (Inclusion) The union of the families generated by all the diameter $d$ cores is the set of all NL trees with diameter $d$.

4. (Nonuniqueness) A diameter $d \mathrm{NL}$ tree may be generated from more than one core. 
Example 3.5. The diameter 5 core (see Figure 1) is the only NL tree on 10 vertices, and there are two diameter 6 cores (see Figure 2). We display the six diameter 7 cores, up to isomorphism.
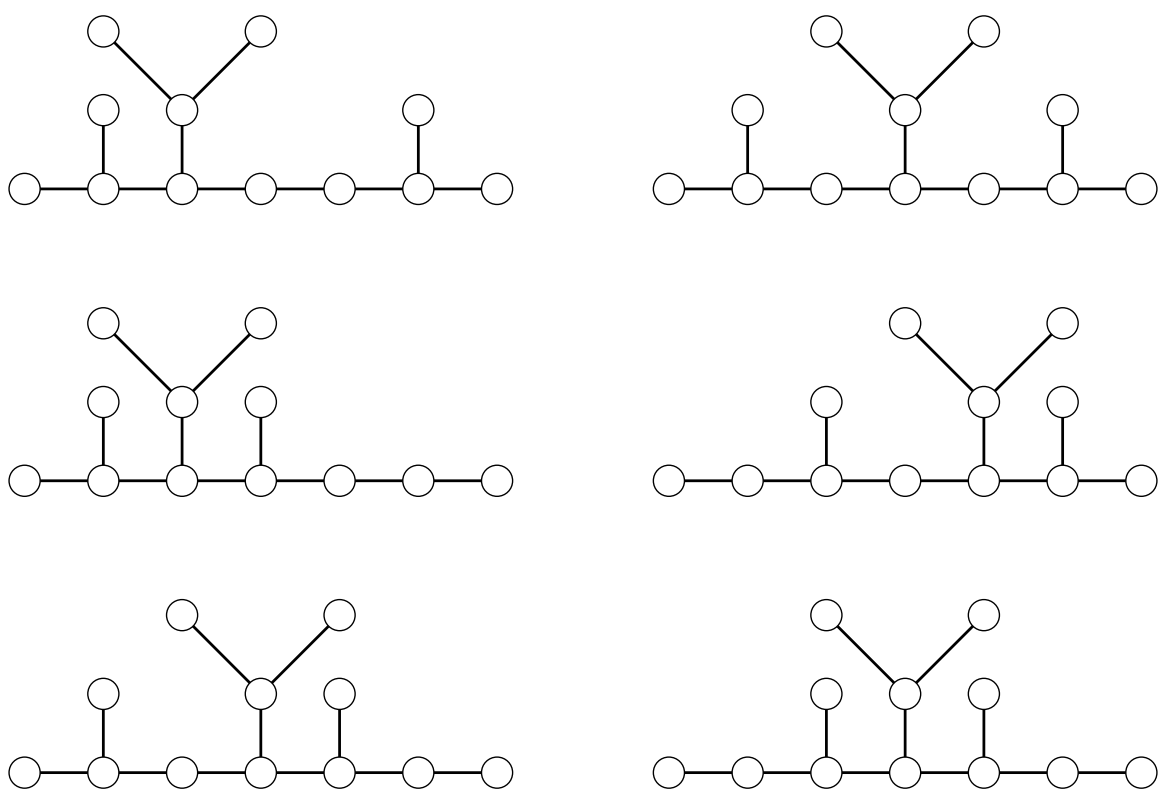

\subsection{Counting Non-isomorphic Diameter $d$ Cores}

We first introduce an algorithm for generating diameter $d$ cores.

Algorithm 3.6. We start with the 10-vertex NL tree (Figure 1) By the minimality of cores and Proposition 3.3, diameter $d$ cores are obtained by adding $d-5$ vertices to the 10-vertex NL tree in a way that the addition of each vertex increases the diameter by 1 , which are, in fact, $d-5$ edge subdivisions on exactly one diameter. Since the diameter is 5 , generating cores involves edge subdivisions on four edges. Let $p, q, r, s$ denote the number of edge subdivisions performed on each of these four edges. Then, $(p, q, r, s)$, an ordered partition of $d-5$ into four parts, each of which is a nonnegative integer ( 0 is allowed), represents a diameter $d$ core that is obtained by subdividing the first edge of one diameter in the smallest NL tree $p$ times, the second $q$ times, the third $r$ times, and the last $s$ times. Thus, the set of all diameter $d$ cores equals all such ordered partitions of $d-5$ into four parts.

Example 3.7. We revisit the six diameter 7 cores in Example 3.5. By Algorithm 3.6, we consider ordered partitions of $d-5=7-5=2$. From upper left to lower right, the cores correspond to partitions $(0,0,2,0)$, $(0,1,1,0),(0,0,0,2),(1,1,0,0),(0,1,0,1)$, and $(1,0,1,0)$. One might wonder if some partitions such as $(2,0,0,0)$ are missing. The answer is no because of forward/backward symmetry, which results from orienting the diameter from either end.

Proposition 3.8. Diameter $d$ cores with ordered partitions $(p, q, r, s)$ and $(s, r, q, p)$ are isomorphic.

By Remark 3.4, diameter $d$ cores are finite for each $d$, so a natural question to ask is the number of distinct diameter $d$ cores. We define $\mathcal{C}(d)$ to be the collection of distinct diameter $d$ cores. And $|\mathcal{C}(d)|$ denotes the number of non-isomorphic diameter $d$ cores.

\section{Proposition 3.9.}

$$
|\mathcal{C}(d)|=\left\{\begin{array}{l}
\frac{\left(\begin{array}{c}
d-2 \\
3
\end{array}\right)}{2}, \text { if d is even, } \\
\frac{\left(\begin{array}{c}
-2 \\
3
\end{array}\right)}{2}+\frac{d-3}{4}, \text { if } d \text { is odd. }
\end{array}\right.
$$


Proof. Among the $d$ vertices along a diameter, we choose any subset of three vertices among the $d-2$ interior vertices of this diameter. Each such selection corresponds to a diameter $d$ core: by considering this subset $\{i, j, k\} \subseteq\{1,2, \ldots, d-2\}$ in the order $i<j<k$, we attach pendent vertices at each of the vertices corresponding to $i$ and $k$ and attach a 3 -path $\left(v_{0}, v_{1}, v_{2}\right)$ to vertex $j$ at $v_{1}$. This generates every diameter $d$ core, but up to isomorphism - we have overcounted. If the diameter is oriented horizontally, two diameter $d$ cores constructed this way will be isomorphic if and only if we obtain one from the other by the vertical reflection through the center of the diameter. When $d$ is even, there is no central vertex, and we have thus counted diameter $d$ cores in pairs; hence there are exactly $\left(\begin{array}{c}d-2 \\ 3\end{array}\right) / 2$ diameter $d$ cores. When $d$ is odd, there is a central vertex in the diameter $d$ path, and there are thus diameter $d$ cores that are automorphic under the vertical reflection. However, in this case, using the notation above, $j$ must be the middle vertex, and the choice of $i$ determines the choice of $k$. There are $(d-3) / 2$ choices for $i$, and so there are exactly $(d-3) / 2$ diameter $d$ cores that are fixed by the vertical reflection through the center of the longest path, meaning $\left(\begin{array}{c}d-2 \\ 3\end{array}\right) / 2$ undercounts $|\mathfrak{C}(d)|$ by exactly $((d-3) / 2) / 2$. The result follows.

We calculate $|\mathcal{C}(d)|$ up to $d=20$, and interestingly, we notice that the first differences of this sequence are increasing repeated squares.

\begin{tabular}{c|c|c|c|c|c|c|c|c|c|c|c|c|c|c|c|c|}
\hline$d$ & 5 & 6 & 7 & 8 & 9 & 10 & 11 & 12 & 13 & 14 & 15 & 16 & 17 & 18 & 19 & 20 \\
\hline \hline$|\mathcal{C}(d)|$ & 1 & 2 & 6 & 10 & 19 & 28 & 44 & 60 & 85 & 110 & 146 & 182 & 231 & 280 & 344 & 408 \\
\hline $\mathcal{C}(d)|-| \mathcal{C}(d-1) \mid$ & 1 & 1 & 4 & 4 & 9 & 9 & 16 & 16 & 25 & 25 & 36 & 36 & 49 & 49 & 64 & 64 \\
\hline
\end{tabular}

Remark 3.10.

$$
|\mathfrak{C}(d+1)|-|\mathfrak{C}(d)|=\left\{\begin{array}{l}
\left(\frac{1}{2}(d-2)\right)^{2}, \text { if } d \text { is even, } \\
\left(\frac{1}{2}(d-3)\right)^{2}, \text { if } d \text { is odd }
\end{array}\right.
$$

In fact, this sequence of $|\mathcal{C}(d)|$ has appeared elsewhere and been recorded in the On-Line Encyclopedia of Integer Sequence [14] with a generating function

$$
\frac{\left(1+k^{2}\right)}{(1-k)^{2}\left(1-k^{2}\right)^{2}} \quad \text { where } k=d-5 \text {. }
$$

Proposition 3.9 provides an explicit formula for this sequence, and observation about first differences in Remark 3.10 can also be additional to the encyclopedia.

\subsection{Diameters in a Core}

We may also ask how many distinct diameters lie in a given core. We identify cores with different number of diameters and determine the number of diameter $d$ cores with a certain number of diameters. Proof details are in Appendix A.

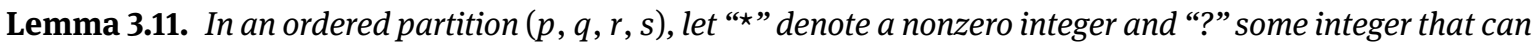
be zero or nonzero. Then,

\begin{tabular}{c|c|c|c|c}
\hline Cores with ordered partition & $\left(\star, ?, ?,{ }^{\star}\right)$ & $\left(0,{ }^{\star}, ?,{ }^{\star}\right)$ & $(\star,,, 0,0)$ or $\left(0,{ }^{\star}, \star, 0\right)$ & $(0, \star, 0,0)$ \\
\hline The number of distinct diameters & 1 & 2 & 4 & 8 \\
\hline
\end{tabular}

Corollary 3.12. For each diameter $d$ core $(d \geqslant 6)$, the number of distinct diameters is a member of $\{1,2,4,8\}$; moreover, among all the diameter $d$ cores $(d \geqslant 7)$, each of $\{1,2,4,8\}$ will occur for at least one of the cores.

Corollary 3.13. In $\mathrm{C}(d)$ with $d \geqslant 7,|\mathcal{C}(d-2)|$ cores have a unique diameter, $\left(\begin{array}{c}d-6 \\ 2\end{array}\right)$ cores have two diameters, 1 core has eight diameters, and the number of cores with four diameters is

$$
\left\{\begin{array}{l}
1.5 d-7.5 \text { if } d \text { is odd, } \\
1.5 d-8 \text { if } d \text { is even. }
\end{array}\right.
$$




\section{Cores and $U(T)$}

After laying the groundwork for cores and exploring their properties, we now discuss $U(T)$ for cores and NL trees.

\subsection{Diameter 5 and 6 NL Trees}

In this subsection, through an extensive examination, we find that for any diameter 5 or $6 \mathrm{NL}$ tree, $U(T)=2$. The proof is based on expansion of cores and eigenvalue assignment.

Lemma 4.1. Suppose $\mathcal{A}$ is a realizable eigenvalue assignment for a tree $T$, and the multiplicity list realized by $\mathcal{A}$ achieves $U(T)$, then for the tree $T^{\prime}$ resulting from adding a pendent vertex at a Parter vertex for some multiple eigenvalue, $U\left(T^{\prime}\right) \leqslant U(T)$.

Proof. Suppose a pendent vertex $u$ is added to a Parter vertex $v$ for $\lambda$ in $\mathcal{A}$ for $T$, which results in $T^{\prime}$. Then, in eigenvalue assignment $\mathcal{A}^{\prime}$ for $T^{\prime}$, we assign $\lambda$ to $u$ and adopt assignment $\mathcal{A}$ for the rest. Notice that besides the assignment of $\lambda$ to the new vertex $u, \mathcal{A}^{\prime}$ slightly differs from $\mathcal{A}$ because for other eigenvalues, some subtree need to contain $u$ in $\mathcal{A}^{\prime}$. However, since $T^{\prime}$ is larger than $T$, there is no worry about overloading for $\mathcal{A}^{\prime}$. Thus, the realizability of $\mathcal{A}$ implies that for $\mathcal{A}^{\prime}$; so, as no new multiplicity 1 eigenvalue is created by the addition of $u, U\left(T^{\prime}\right) \leqslant U(T)$, as desired. $U\left(T^{\prime}\right)<U(T)$ happens when a multiplicity 1 eigenvalue becomes multiplicity 2 as a result of the addition; on the other hand, $U(T)$ remains the same when the multiplicity of a multiple eigenvalue increases by 1 .

Theorem 4.2. If $T$ is a diameter $5 N L$ tree, then $U(T)=2$.

Proof. The 10-vertex NL tree $T_{0}$ (see labeling in Figure 1) is the only diameter 5 core, so by Remark 3.4, any diameter $5 \mathrm{NL}$ tree $T$ can be obtained from $T_{0}$ via pendent vertex addition. Due to the restriction on diameter, no vertex is more than two edges away from $c$. So, to expand $T_{0}$, the choice of places for vertex addition is limited. In particular, at $c$, we can add pendent vertices, 2-paths, or simple stars, whereas we can only add pendent vertices to $v_{i}$.

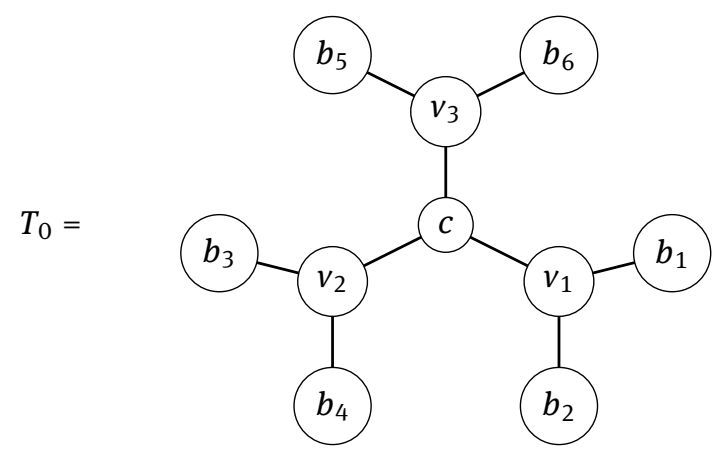

Figure 1: Diameter 5 core

It is known from the database that $(4,2,2,1,1) \in \mathcal{L}\left(T_{0}\right)$, hence $U\left(T_{0}\right)=2$. Call the multiple eigenvalues $\lambda, \alpha$, and $\beta$; an eigenvalue assignment for multiplicity list $(4,2,2,1,1)$ is as follows.

$\mathcal{A}_{1}=\left\{\left\{b_{1}\right\},\left\{b_{2}\right\},\left\{b_{3}\right\},\left\{b_{4}\right\},\left\{b_{5}\right\},\left\{b_{6}\right\},\{c\}\right\}$ with $V_{1}=\left\{v_{1}, v_{2}, v_{3}\right\}$ for $\lambda$.

$\mathcal{A}_{2}=\mathcal{A}_{3}=\left\{\left\{v_{1}, b_{1}, b_{2}\right\},\left\{v_{2}, b_{3}, b_{4}\right\},\left\{v_{3}, b_{5}, b_{6}\right\}\right\}$ with $V_{2}=V_{3}=\{c\}$ for $\alpha$ and $\beta$. 
We will show that as we obtain any diameter $5 \mathrm{NL}$ tree $T$ from $T_{0}$, two 1's in this multiplicity list $(4,2,2,1,1)$ can be maintained by increasing the multiplicity of multiple eigenvalues; therefore, $U(T)=2$. Although diameter $5 \mathrm{NL}$ trees are infinite, there are only four basic ways to add vertices, and any diameter 5 NL trees can be viewed as adding vertices to $T_{0}$ via a combination of these four ways.

The first and second way is about adding one additional vertex to $T_{0}$. We can only do so at either $c$ or some $v_{i}$. Since $c$ and $v_{i}$ are all Parter vertices for some multiple eigenvalue, i.e. $\left\{c, v_{1}, v_{2}, v_{3}\right\} \subseteq V_{1} \cup V_{2} \cup V_{3}$, by Lemma 4.1, trees resulting from such vertex addition have $U(T)=2$. The third way is to add a 2-path to $T_{0}$, say $\left\{u_{1}, u_{2}\right\}$, which has to be adjacent to $c$. Then, because $V_{2}=V_{3}=\{c\}$ for $\alpha$ and $\beta$, we assign $\alpha$ and $\beta$ to this 2-path. As a result, $(4,3,3,1,1)$ is realized. The fourth way involves adding a simple star to $T_{0}$, which again, requires the center of the star to be adjacent to $c$. We may assume adding a (degenerate) simple star, i.e. a 3-path $\left\{u_{2}, u_{1}, u_{3}\right\}$ with $u_{1}$ adjacent to $c$ to $T_{0}$. This suffices because if $u_{1}$ is a Parter vertex for some multiple eigenvalue in this case, Lemma 4.1 also covers stars with more pendent vertices. Multiplicity list $(5,3,3,1,1)$ can be realized by the following eigenvalue assignment.

$\mathcal{A}_{1}=\left\{\left\{b_{1}\right\},\left\{b_{2}\right\},\left\{b_{3}\right\},\left\{b_{4}\right\},\left\{b_{5}\right\},\left\{b_{6}\right\},\{c\},\left\{u_{2}\right\},\left\{u_{3}\right\}\right\}$ with $V_{1}=\left\{v_{1}, v_{2}, v_{3}, u_{1}\right\}$ for $\lambda$.

$\mathcal{A}_{2}=\mathcal{A}_{3}=\left\{\left\{v_{1}, b_{1}, b_{2}\right\},\left\{v_{2}, b_{3}, b_{4}\right\},\left\{v_{3}, b_{5}, b_{6}\right\},\left\{u_{1}, u_{2}, u_{3}\right\}\right\}$ with $V_{2}=V_{3}=\{c\}$ for $\alpha$ and $\beta$.

Any diameter $5 \mathrm{NL}$ tree $T$ can be dissected into a combination of these four ways of vertex addition to the diameter 5 core $T_{0}$; since eigenvalue assignments in these four ways do not conflict with one another, $U(T)=2$.

Theorem 4.3. If $T$ is a diameter $6 N L$ tree, then $U(T)=2$.

Proof. The idea is similar to that for Theorem 4.2, i.e. no new vertex is more than two edges away from any vertex in a diameter 6 core, although the examination is more complicated. There are two diameter 6 cores, $T_{1}$ and $T_{2}$, and we label their vertices as in Figure 2. $U\left(T_{1}\right)=U\left(T_{2}\right)=2$ because the multiplicity list $(3,2,2,2,1,1)$ is realizable for both of them, according to the database. Call the multiple eigenvalues $\lambda, \alpha$, $\beta$, and $\gamma$. We display the eigenvalue assignment for $T_{1}$ and $T_{2}$.
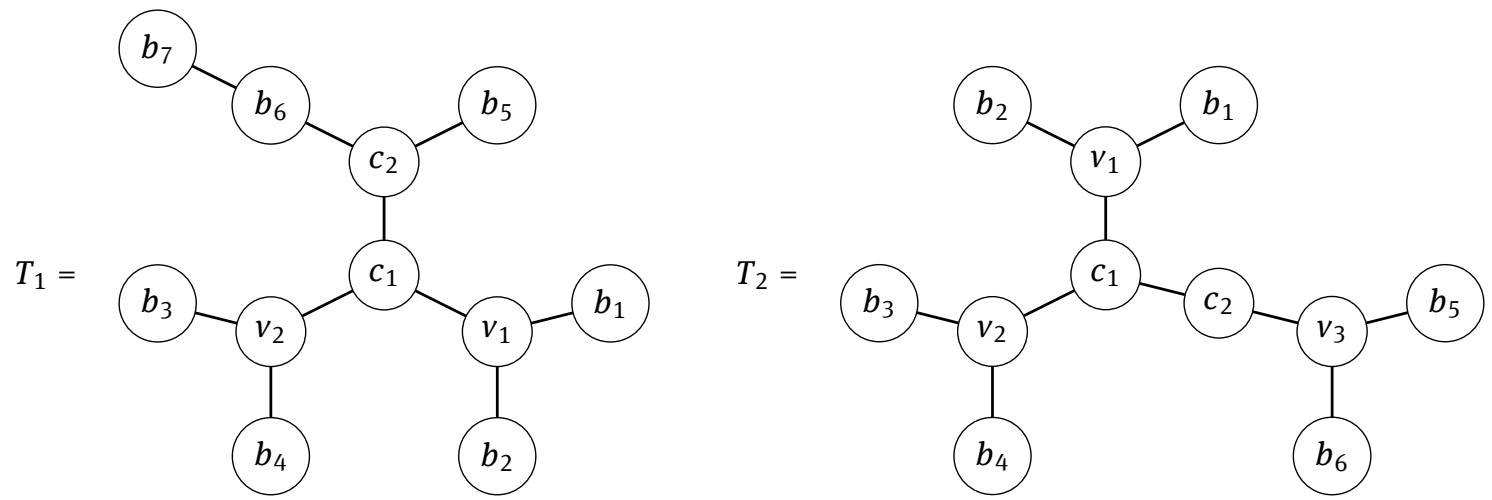

Figure 2: Diameter 6 cores

For $T_{1}: \mathcal{A}_{1}=\left\{\left\{b_{1}\right\},\left\{b_{2}\right\},\left\{b_{3}\right\},\left\{b_{4}\right\},\left\{c_{1}, c_{2}, b_{5}, b_{6}, b_{7}\right\}\right\}$ with $V_{1}=\left\{v_{1}, v_{2}\right\}$ for $\lambda$. $\mathcal{A}_{2}=\mathcal{A}_{3}=\left\{\left\{v_{1}, b_{1}, b_{2}\right\},\left\{v_{2}, b_{3}, b_{4}\right\},\left\{c_{2}, b_{5}, b_{6}, b_{7}\right\}\right\}$ with $V_{2}=V_{3}=\left\{c_{1}\right\}$ for $\alpha$ and $\beta$. $\mathcal{A}_{4}=\left\{\left\{b_{5}\right\},\left\{b_{6}, b_{7}\right\},\left\{c_{1}, v_{1}, b_{1}, b_{2}, v_{2}, b_{3}, b_{4}\right\}\right\}$ with $V_{4}=\left\{c_{2}\right\}$ for $\gamma$.

For $T_{2}: \mathcal{A}_{1}=\left\{\left\{b_{1}\right\},\left\{b_{2}\right\},\left\{b_{3}\right\},\left\{b_{4}\right\},\left\{c_{1}\right\},\left\{v_{3}, b_{5}, b_{6}\right\}\right\}$ with $V_{1}=\left\{v_{1}, v_{2}, c_{2}\right\}$ for $\lambda$. $\mathcal{A}_{2}=\mathcal{A}_{3}=\left\{\left\{v_{1}, b_{1}, b_{2}\right\},\left\{v_{2}, b_{3}, b_{4}\right\},\left\{c_{2}, b_{5}, b_{6}, v_{3}\right\}\right\}$ with $V_{2}=V_{3}=\left\{c_{1}\right\}$ for $\alpha$ and $\beta$. $\mathcal{A}_{4}=\left\{\left\{b_{5}\right\},\left\{b_{6}\right\},\left\{c_{1}, c_{2}, v_{1}, b_{1}, b_{2}, v_{2}, b_{3}, b_{4}\right\}\right\}$ with $V_{4}=\left\{v_{3}\right\}$ for $\gamma$.

Any diameter $6 \mathrm{NL}$ tree $T$ can be obtained from $T_{1}$ or $T_{2}$ by a sequence of pendent vertex addition. We will again show that this multiplicity list $(3,2,2,2,1,1)$ can be updated as we obtain $T$ from $T_{1}$ or $T_{2}$ so that $U(T)=2$. We explain the eigenvalue assignment strategy for all basic ways to expand $T_{1}$ and $T_{2}$. 
First, for $T_{1}$, there are five places to add one additional vertex and two places to add a 2-path or a star. To add one additional vertex to $T_{1}$, we can do so at $c_{1}, v_{1}\left(v_{2}\right), c_{2}, b_{5}$, or $b_{6}$. Because $c_{1}, v_{1}\left(v_{2}\right)$, and $c_{2}$ are all Parter vertices for some multiple eigenvalue in $T_{1}$, Lemma 4.1 applies to these three cases. The only remaining cases are to add a pendent vertex at $b_{5}$ or $b_{6}$.

1. Adding a pendent vertex $u$ to $b_{5}$ : we can increase $m(\lambda)$ so as to realize $(4,2,2,2,1,1)$. Specifically, an eigenvalue assignment can be as follows:

$\mathcal{A}_{1}=\left\{\left\{b_{1}\right\},\left\{b_{2}\right\},\left\{b_{3}\right\},\left\{b_{4}\right\},\left\{c_{1}\right\},\left\{b_{5}, u\right\},\left\{b_{6}, b_{7}\right\}\right\}$ with $V_{1}=\left\{v_{1}, v_{2}, c_{2}\right\}$ for $\lambda$. $\mathcal{A}_{2}=\mathcal{A}_{3}=\left\{\left\{v_{1}, b_{1}, b_{2}\right\},\left\{v_{2}, b_{3}, b_{4}\right\},\left\{c_{2}, b_{5}, u, b_{6}, b_{7}\right\}\right\}$ with $V_{2}=V_{3}=\left\{c_{1}\right\}$ for $\alpha$ and $\beta$. $\mathcal{A}_{4}=\left\{\left\{b_{5}, u\right\},\left\{b_{6}, b_{7}\right\},\left\{c_{1}, v_{1}, b_{1}, b_{2}, v_{2}, b_{3}, b_{4}\right\}\right\}$ with $V_{4}=\left\{c_{2}\right\}$ for $\gamma$.

2. Adding a pendent vertex $u$ to $b_{6}$ : we can again increase $m(\lambda)$ so as to realize $(4,2,2,2,1,1)$, but via a different eigenvalue assignment:

$\mathcal{A}_{1}=\left\{\left\{b_{1}\right\},\left\{b_{2}\right\},\left\{b_{3}\right\},\left\{b_{4}\right\},\left\{c_{1}\right\},\left\{b_{5}\right\},\left\{b_{6}, b_{7}, u\right\}\right\}$ with $V_{1}=\left\{v_{1}, v_{2}, c_{2}\right\}$ for $\lambda$. $\mathcal{A}_{2}=\mathcal{A}_{3}=\left\{\left\{v_{1}, b_{1}, b_{2}\right\},\left\{v_{2}, b_{3}, b_{4}\right\},\left\{c_{2}, b_{5}, u, b_{6}, b_{7}\right\}\right\}$ with $V_{2}=V_{3}=\left\{c_{1}\right\}$ for $\alpha$ and $\beta$. $\mathcal{A}_{4}=\left\{\{u\},\left\{b_{7}\right\},\left\{c_{1}, c_{2}, b_{5}, v_{1}, b_{1}, b_{2}, v_{2}, b_{3}, b_{4}\right\}\right\}$ with $V_{4}=\left\{b_{6}\right\}$ for $\gamma$.

Beyond the addition of pendent vertices, we can add a 2-path or a star at either $c_{1}$ or $c_{2}$ in $T_{1}$. On the one hand, since $c_{1}$ is a Parter vertex for both $\alpha$ and $\beta$, just like $c$ in Theorem 4.2, a similar strategy for eigenvalue assignment when a 2-path or a simple star is added can be adopted. On the other hand, $c_{2}$ 's situation is slightly different because $c_{2}$ is only a Parter vertex for $\gamma$. First, a tree $T$ resulting from adding a 2-path at $c_{2}$ is the same as adding a pendent vertex at $b_{5}$ and another at $c_{2}$. By the discussion above and Lemma 4.1, $U(T)=2$. Second, adding a degenerate star (i.e., a 3-path, which suffices for any star together with Lemma 4.1) at $c_{2}$ is equivalent to adding a pendent vertex at $b_{6}$ and a 2-path at $c_{2}$. Then, again, $U(T)=2$ by previous results. This completes the discussion about all the basic ways of expanding the diameter 6 core $T_{1}$.

Now, for the other diameter 6 core, $T_{2}$, pendent vertices, 2-paths, and stars can be added. One additional pendent vertex can be added at any nonpendent vertex, and in the eigenvalue assignment for $T_{2}$, all of them are a Parter vertex for some multiple eigenvalue, so Lemma 4.1 applies. Then, again, 2-paths and stars can only be adjacent to $c_{1}$ or $c_{2}$. First, $c_{1}$ in $T_{2}$ is similar to that in $T_{1}$ with regard to its role as a Parter vertex for two multiple eigenvalues in the assignment, so identical strategy is adopted here. Second, when a 2-path is added to $c_{2}$ in $T_{2}$, it is the same as adding a pendent vertex at $b_{5}$ and another at $b_{6}$ in $T_{1}$. Both additions maintain that $U(T)=2$. Moreover, the tree resulting from the addition of a degenerate star at $c_{2}$ can also be obtained by adding two pendent vertices at $b_{5}$ and one pendent vertex at $b_{6}$ in $T_{1}$. So, by previous results on $T_{1}$, we conclude $U(T)$ remain 2 here as well.

We have shown what eigenvalue assignment is like for each basic way of vertex addition to both cores, and any diameter $6 \mathrm{NL}$ tree is obtained by a combination of these. Thus, they all have a multiplicity list without more 1's than their core. So, $U(T)=2$ for any diameter $6 \mathrm{NL}$ tree.

When the diameter of an NL tree is as small as 5 or 6 , the structure of the tree is greatly limited, despite the fact that there are infinitely many diameter 5 and 6 NL trees. We suspect that $U(T)=2$ holds true for all diameter 7 NL trees as well; no counterexample is known, but a similar proof to that of 5 or 6 appears complicated. This conjecture is formally stated in Section 4.2, in a more general form for any diameter $d$ NL tree. (However, it is certain that not every diameter $8 \mathrm{NL}$ tree has $U(T)=2$.)

\section{2 $U(T)$ for diameter $d$ cores}

We employ the Implicit function theorem (IFT) approach developed in [11] whose purpose is to show a certain multiplicity list is in the catalog of a given tree.

Theorem 4.4. (Implicit function theorem) Let $f: \mathbb{R}^{n+m} \rightarrow \mathbb{R}^{n}$ be a continuously differentiable function. Suppose that, for $x_{0} \in \mathbb{R}^{n}$ and $y_{0} \in \mathbb{R}^{m}, f\left(x_{0}, y_{0}\right)=0$ and the Jacobian $\frac{\partial f}{\partial x}\left(x_{0}, y_{0}\right)$ is invertible. Then there exists a neighborhood $U \subset \mathbb{R}^{m}$ around $y_{0}$ such that $f(x, y)=0$ has a solution $x$ for any fixed $y \in U$. Furthermore, regarding the solution $x$ as a function of $y$, gives a function continuous at $\left(x_{0}, y_{0}\right)$. 
We think of a matrix with graph $G$ as a multivariable function of the entries. Then we build an "initial matrix" $A^{(0)}$ whose graph $G_{0}$ is a subgraph of $G$ by edge containment such that the spectrum of $A^{(0)}$ has the multiplicities we desire. This involves specification of a number of determinant conditions (characteristic polynomials). We need to choose as many variables called implicit entries as the conditions and obtain a nonsingular Jacobian with respect to these entries. Then we can perturb the edges in $G \backslash G_{0}$ by some sufficiently small $\epsilon \neq 0$. By letting $F$ be a sufficient set of determinant conditions for the multiplicities, the resulting matrix will have graph $G$ but the same spectrum as $A^{(0)}$. More details can be found in [11].

Theorem 4.5. Let $G$ be a graph, let $F=\left(f_{k}\right)$ be a vector of $r$ determinant conditions and designate $r$ entries as implicit entries. Suppose a real symmetric matrix $A^{(0)}$ is the direct sum of irreducible matrices $A_{1}, A_{2}, \ldots, A_{p}$, and

1. $F\left(A^{(0)}\right)=0$;

2. $\mathrm{G}\left(A^{(0)}\right)$ is a subgraph of $G$;

3. if $a_{i j}^{(0)}=0, i \neq j$, then $(i, j)$ is not an implicit entry; and

4. $\operatorname{det} J\left(A^{(0)}\right)$, the Jacobian of $F$ with respect to the implicit entries, evaluated at $A^{(0)}$, is nonzero.

Then there exists a matrix $A \in \mathcal{S}(G)$ such that $F(A)=0$ and the ordered multiplicities of $A$ are a refinement of the ordered multiplicities of $A^{(0)}$.

The following two lemmas in [11] are helpful with checking condition 4 in Theorem 4.5, using reducibility of $A^{(0)}$. The first one is general, whereas the second addresses a special case in which $A^{(0)}$ is diagonal.

Lemma 4.6. Let $T$ be a tree and $F=\left(f_{k}\right)_{k=1, \ldots, r}$ be defined as above, with $r$ implicit entries identified. Suppose that a real symmetric matrix $A^{(0)}$, whose graph is a subgraph of $T$, is the direct sum of irreducible matrices $A_{1}^{(0)}, A_{2}^{(0)}, \ldots, A_{p}^{(0)}$. Let $J\left(A^{(0)}\right)$ be the Jacobian matrix of $F$ with respect to the implicit entries evaluated at $A^{(0)}$, and suppose that

1. every off-diagonal implicit entry in $A^{(0)}$ has a non-zero value;

2. for every $k=1, \ldots, r, f_{k}\left(A_{l}^{(0)}\right)=0$ for precisely one $l \in\{1, \ldots, p\}$; and

3. for every $l=1, \ldots, p$, the columns of $J\left(A^{(0)}\right)$ associated with the implicit entries of $A_{l}^{(0)}$ are linearly independent.

Then $J\left(A^{(0)}\right)$ is nonsingular.

Lemma 4.7. Let $F=\left(f_{k}\right)$ be a vector of $r$ determinant conditions, and let $A^{(0)}$ be a diagonal matrix. Suppose that for every $k=1, \ldots, r, f_{k}\left(A^{(0)}[l]\right)=0$ for precisely one $l \in\{1, \ldots, n\}$. Take $a_{l l}$ to be an implicit entry if and only if $f_{k}\left(A^{(0)}[l]\right)=0$ for some $k$. If there are then $r$ implicit entries, the Jacobian of $F$ with respect to the implicit entries evaluated at $A^{(0)}$ is nonzero.

Theorem 4.8. If $T$ is a diameter $d$ core, then

$$
U(T)=\left\{\begin{array}{ll}
2 & \text { if } d<7 \\
d-5 & \text { if } d \geqslant 7
\end{array} .\right.
$$

Proof. If $T$ is a diameter $d$ core such that $d<7$, then either $d=5$ or $d=6$. By Theorem 4.2 and 4.3, $U(T)=2$. Now, suppose $T$ is a diameter $d$ core such that $d \geqslant 7$ with ordered partition $(p, q, r, s)$, then $p+q+r+s \geqslant 2$. We label vertices in a core as follows. Vertices $p, q, r$, and $s$ are placeholders for paths with length $p, q$, $r$, and $s$. 


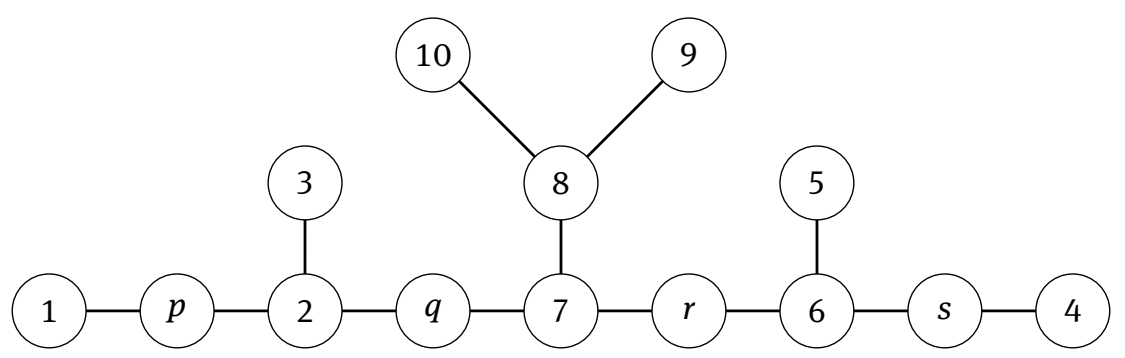

To prove $U(T)=d-5$, since we already know $U(T) \geqslant 2 d-n=d-5$, for the reverse inequality, it suffices to show that the multiplicity list $\left(2,2,2,2,2,1^{d-5}\right) \in \mathcal{L}(T)$. Indeed, $\left(2,2,2,2,2,1^{d-5}\right)$ is the only multiplicity list with this many 1's. We will construct an $A=\left(a_{i j}\right) \in \mathcal{S}(T)$ with the five distinct multiplicity 2 eigenvalues being $\alpha, \lambda, \beta, \tau$, and $\gamma$. Sufficient conditions for having $m_{A}(\alpha)=m_{A}(\lambda)=m_{A}(\beta)=m_{A}(\tau)=m_{A}(\gamma)=2$ can be specified as a vector-valued function. Let $F=\left(f_{k}\right)_{k=1, \ldots, 15}$ be

$$
\begin{aligned}
f_{1}=\operatorname{det}\left(A[1, p]-\alpha I_{1+p}\right) & =0 \\
f_{2}=a_{3,3}-\alpha & =0 \\
f_{3}=\operatorname{det}\left(A[4,5,6,7,8,9,10, q, r, s]-\alpha I_{7+q+r+s}\right) & =0 \\
f_{4}=\operatorname{det}\left(A[4, s]-\lambda I_{1+s}\right) & =0 \\
f_{5}=a_{5,5}-\lambda & =0 \\
f_{6}=\operatorname{det}\left(A[1,2,3,7,8,9,10, p, q, r]-\lambda I_{7+p+q+r}\right) & =0 \\
f_{7}=a_{9,9}-\beta & =0 \\
f_{8}=a_{10,10}-\beta & =0 \\
f_{9}=\operatorname{det}\left(A[1,2,3,4,5,6,7, p, q, r, s]-\beta I_{7+p+q+\gamma+s}\right) & =0 \\
f_{10}=\operatorname{det}\left(A[1,2,3, p, q]-\tau I_{3+p+q}\right) & =0 \\
f_{11}=\operatorname{det}\left(A[4,5,6, r, s]-\tau I_{3+r+s}\right) & =0 \\
f_{12}=\operatorname{det}\left(A[8,9,10]-\tau I_{3}\right) & =0 \\
f_{13}=\operatorname{det}\left(A[1,2,3, p, q]-\gamma I_{3+p+q}\right) & =0 \\
f_{14}=\operatorname{det}\left(A[4,5,6, r, s]-\gamma I_{3+r+s}\right) & =0 \\
f_{15}=\operatorname{det}\left(A[8,9,10]-\gamma I_{3}\right) & =0 .
\end{aligned}
$$

Any matrix in the kernel of $F$ has the desired multiplicity list. Now we construct a real symmetric matrix $A^{(0)}=\left(a_{i j}^{(0)}\right)$ with $F\left(A^{(0)}\right)=0$ whose graph is a subgraph of $T$. First of all, some constraints of $F$ on $A^{(0)}$ are straightforward, such as $a_{3,3}^{(0)}=\alpha, a_{5,5}^{(0)}=\lambda, a_{9,9}^{(0)}=\beta$, and $a_{10,10}^{(0)}=\beta$ by $f_{2}, f_{5}, f_{7}$, and $f_{8}$. Moreover, subject to $f_{7}, f_{8}, f_{12}$, and $f_{15}=0$, the three eigenvalues in $A^{(0)}[8,9,10]$ have to be $\beta, \tau$, and $\gamma$. As a result, the submatrix $A[8,9,10]$ is determined, up to a fixed sum of squares of the off-diagonal entries.

$$
A[8,9,10]=\left[\begin{array}{ccc}
\tau+\gamma-\beta & a_{8,9} & a_{8,10} \\
a_{8,9} & \beta & 0 \\
a_{8,10} & 0 & \beta
\end{array}\right] \text { where } a_{8,9}^{2}+a_{8,10}^{2}=\beta(\tau+\gamma-\beta)-\tau \gamma
$$

Thus, we may ignore this branch in $T$ and the corresponding 3-by-3 submatrix in $A^{(0)}$. What remains to be considered are conditions $F \backslash\left\{f_{7}, f_{8}, f_{12}, f_{15}\right\}$. Similarly, when $p=q=0$ or $r=s=0$, the corresponding branch and 3-by-3 submatrix will also be determined with eigenvalues $\{\alpha, \tau, \gamma\}$ or $\{\lambda, \tau, \gamma\}$. Therefore, without loss of generality, we may only consider and construct a submatrix of $A^{(0)}$ whose underlying graph does not have a fully determined branch.

On the one hand, when (i) $d \geqslant 9$, or (ii) $d=8$ and either $p=q=0$ or $r=s=$ 0 , we may choose the initial matrix $A^{(0)}$ to be diagonal, satisfying the conditions in $F$ subject to Lemma 4.7. For example, in the case when $p=1, q=1, r=1$, and $s=1$, we may choose 
$A^{(0)}[1,2,3,4,5,6,7, p, q, r, s]=\operatorname{diag}(\alpha, \gamma, \alpha, \lambda, \lambda, \tau, \beta, \tau, \lambda, \alpha, \gamma)$. By Lemma 4.7, $J\left(A^{(0)}\right)$ is nonsingular; hence, by Theorem 4.5, $\left(2,2,2,2,2,1^{d-5}\right) \in \mathcal{L}(T)$ as desired.

For diameter 7 and 8 cores that do not meet the above criterion, we have to choose some off-diagonal entries to be implicit entries. Besides the use of diagonal entries for implicit entries, one or two pairs of offdiagonals entries are needed, depending on the core being considered. We can choose from the off-diagonal entries corresponding to edges $(2,3)$ and $(5,6)$. Then we construct $A^{(0)}$ in which the 2-by-2 block consisting of vertices 2 and 3 (respectively, vertices 5 and 6) has eigenvalues $\tau$ and $\gamma$, i.e. $a_{3,3}^{(0)}=\alpha, a_{2,2}^{(0)}=\tau+\gamma-\alpha$, and $a_{2,3}^{(0)}=a_{3,2}^{(0)}=\sqrt{(\tau-\alpha)(\alpha-\gamma)} \neq 0$ (respectively, $a_{5,5}^{(0)}=\lambda, a_{6,6}^{(0)}=\tau+\gamma-\lambda$, and $a_{5,6}^{(0)}=a_{6,5}^{(0)}=\sqrt{(\tau-\lambda)(\lambda-\gamma)}$.) Then, by the formulas for entries in the Jacobian provided in [11], the columns of $J\left(A^{(0)}\right)$ associated with these implicit entries are linearly independent because $\tau$ and $\gamma$ are distinct. Now, by Lemma 4.6, $J\left(A^{(0)}\right)$ is again nonsingular. And hence, by Theorem 4.5, $\left(2,2,2,2,2,1^{d-5}\right) \in \mathcal{L}(T)$ as desired, completing the proof.

Lastly, we make a note about a potential upper bound for $U(T)$ for any diameter $d$ NL tree. Since we know $U(T)$ for cores by Theorem 4.8, it is conjectured that $U(T)$ for a core is an upper bound for all trees in the family generated by this core. If we knew the result for linear trees about the change of $U(T)$ upon vertex addition in [3] for NL trees, then Conjecture 4.9 would follow. Note that $d-5$ is $U(T)$ shared by any diameter $d$ core $T$, from which NL trees of this diameter are built.

Conjecture 4.9. If $T$ is a diameter $d(\geqslant 7)$ NL tree, then $U(T) \leqslant d-5$.

\subsection{Determining the Catalog of Cores}

The catalog for linear trees is characterized by the LSP, but little is known about that for NL trees. However, with the tools we have developed, we know more about cores.

Lemma 4.10. For any diameter $d$ core $T, M(T)=P(T)=4$.

Proof. $M(T)=P(T)$ holds true for any tree [4]. Secondly, for any diameter $d$ core $T$, one path cover consists of three paths that start at a pendent vertex, go through one HDV, and end at a pendent vertex, with length $p+3, s+3$, and 3, and one path that contains the rest with length $q+r+1$. So, $P(T) \leqslant 4$. Also, $P(T) \geqslant 4$ because $T$ results from the 10 -vertex NL tree with path cover number 4 via edge subdivisions, for which the path cover number does not decrease.

Here we cite a prior result in [12] about $M_{2}$, the largest sum of the top two multiplicities over lists in the catalog for $T$.

Theorem 4.11. For any tree $T$ on $n$ vertices, $M_{2}(T) \leqslant n+2-d(T)$.

Theorem 4.12. For any diameter $d(\geqslant 7)$ core $T$,

$$
\begin{aligned}
\mathcal{L}(T) \subseteq\{ & 4221^{d-3}, 421^{d-1}, 41^{d+1}, 3321^{d-3}, 331^{d-1}, 32221^{d-4}, 3221^{d-2}, 321^{d}, 31^{d+2}, \\
& \left.222221^{d-5}, 22221^{d-3}, 2221^{d-1}, 2221^{d-1}, 221^{d+1}, 21^{d+3}, 1^{d+5}\right\} .
\end{aligned}
$$

Moreover, for $T \in \mathcal{C}(d)$ in which no interior edges are subdivided, i.e., the ordered partition into four parts $(p, q, r, s)$ has $q=r=0$,

$$
\begin{aligned}
\mathcal{L}(T) \subseteq & \left\{4221^{d-3}, 421^{d-1}, 41^{d+1}, 32221^{d-4}, 3221^{d-2}, 321^{d}, 31^{d+2},\right. \\
& \left.222221^{d-5}, 22221^{d-3}, 2221^{d-1}, 2221^{d-1}, 221^{d+1}, 21^{d+3}, 1^{d+5}\right\} .
\end{aligned}
$$

Proof. By Lemma 4.10, $M(T)=P(T)=4$, so any multiplicity list containing a multiplicity greater than 4 is not allowed in $\mathcal{L}(T)$. Next, we rule out some candidate multiplicity lists by citing prior results and/or showing 
an eigenvalue assignment is impossible. The following are three prior results we will use. First, by Theorem 4.8, $U(T)=d-5$. Second, the minimum number of distinct eigenvalues in a list is no less than $d$. Third, by Theorem 4.11, the sum of the largest two multiplicities, $M_{2}(T) \leqslant n+2-d(T)=d+5+2-d=7$.

Again, in order to describe eigenvalue assignments more easily, we label vertices in $T$ as follows. Vertices $p, q, r$, and $s$ are placeholders for paths with length $p, q, r$, and $s$, and $p+q+r+s=d-5$.

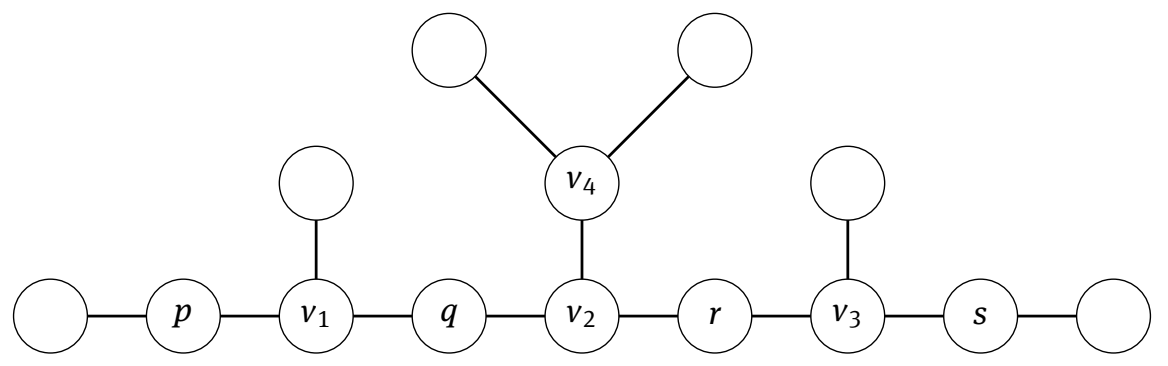

First, we start with multiplicity lists with the maximum multiplicity, namely, 4, in it. Any list containing $(4,4)$ is not possible because $M_{2}=8 \& 7$. Any list containing $(4,3)$ is also ruled out because no assignment produces two eigenvalues with multiplicity 4 and 3 simultaneously. To be specific, the multiplicity 4 eigenvalue, say $\lambda$, requires three nonadjacent HDV's, either $\left\{v_{1}, v_{2}, v_{3}\right\}$ or $\left\{v_{1}, v_{3}, v_{4}\right\}$, as Parter vertices. In the former case, $v_{4}$ or $v_{2}$ can only be a Parter vertex for another eigenvalue with multiplicity at most 2; in the latter case, only $v_{2}$ can be a Parter vertex for up to two eigenvalues with multiplicity at most 2 . Thus, another multiplicity 3 eigenvalue is not allowed, which rules out any list containing $(4,3)$. The list $(4,2,2)$ is indeed allowed, with plausible eigenvalue assignments suggested by previous discussion. However, any list containing $(4,2,2,2)$ is again impossible because in the case when $\left\{v_{1}, v_{2}, v_{3}\right\}$ are Parter vertices for $\lambda$, after using $v_{4}$ as a Parter vertex for a multiplicity 2 eigenvalue, $\alpha$, and $v_{2}$ for another one, $\beta$, the 3-path adjacent to $v_{2}$ containing $v_{4}$ is "full" against overloading as it has $\lambda, \alpha$, and $\beta$ as its three eigenvalues. On the other hand, in the case when $\left\{v_{1}, v_{3}, v_{4}\right\}$ are Parter vertices for $\lambda$, only $v_{2}$ is available for being a Parter vertex for multiplicity 2 eigenvalues, and it can be a Parter vertex for up to two such eigenvalues, say $\alpha$ and $\beta$. A third multiplicity 2 eigenvalue is not allowed because the 3-path is again "full" with $\lambda, \alpha$, and $\beta$.

Second, we consider multiplicity lists containing 3 as the largest multiplicity. Any list containing $(3,3,3)$ is ruled out because an assignment achieving three multiplicity 3 eigenvalues, say $\alpha, \beta$, and $\gamma$, does not exist. Since no vertex has degree more than three, each of $\alpha, \beta$, and $\gamma$ must have at least two nonadjacent Parter vertices. $v_{1}, v_{3}$, and $v_{4}$ can be a Parter vertex for one eigenvalue each because of the limited size of the branches. However, since $v_{2}$ and $v_{4}$ are adjacent, $v_{4}$ has to be a Parter vertex for one of $\alpha, \beta$, and $\gamma$ together with either $v_{1}$ or $v_{3}$. Without loss of generality, suppose $\left\{v_{1}, v_{4}\right\}$ are Parter vertices for $\alpha$, then $v_{3}$ can be a Parter vertex for one of $\beta$ and $\gamma$, only if together with $v_{2}$ when $r \neq 0$, because otherwise $v_{2}$ and $v_{3}$ would not be adjacent. As a result, the last eigenvalue has multiplicity at most 2 with $v_{2}$ being the only possible Parter vertex given $v_{1}, v_{3}$, and $v_{4}$ are fully occupied. Thus, any list containing $(3,3,3)$ is not in $\mathcal{L}(T)$. In addition, although the list $\left(3,3,2,1^{d-3}\right)$ is suggested realizable by the eigenvalue assignment above (only if $q \neq 0$ or $r \neq 0$ ), any list containing $(3,3,2,2)$ is impossible. Again, multiplicity 3 eigenvalues must have at least two nonadjacent Parter vertices. $v_{1}, v_{3}$, and $v_{4}$ can be a Parter vertex for no more than one eigenvalue each. Suppose the most flexible situation when $q \neq 0$ and $r \neq 0$, then for the two multiplicity 3 eigenvalues, say $\alpha$ and $\beta$, the choice of Parter vertices can be (i) $v_{4}$ with one of $v_{1}$ and $v_{3}$ for $\alpha$, and $v_{2}$ with the other of $v_{1}$ and $v_{3}$ for $\beta$, or (ii) $v_{1}$ and $v_{2}$ for $\alpha$, and $v_{3}$ and $v_{2}$ for $\beta$. In both cases, two more multiplicity 2 eigenvalues are impossible. In particular, in the former case, $v_{1}, v_{3}$, and $v_{4}$ are all occupied, and $v_{2}$ can be a Parter vertex for at most one multiplicity 2 eigenvalue because the 3-path adjacent to $v_{2}$ has already had two eigenvalues, $\alpha$ and $\beta$, with $\alpha$ occurring on both pendents and $\beta$ by the fact that $v_{2}$ is a Parter for $\beta$. In the latter case, either $v_{4}$ or $v_{2}$ is available for being a Parter vertex for a multiplicity 2 eigenvalue, but they cannot each be a Parter vertex for two distinct multiplicity 2 eigenvalues. If it happened, the 3-path adjacent to $v_{2}$ would have four eigenvalues assigned to it, which is a contradiction. Therefore, any list containing $(3,3,2,2)$ is ruled out by assignment. Lastly, any list containing $(3,2,2,2,2)$ is not possible because it would contain no more than $n-(3+2+2+2+2)=(d+5)-11=d-6$ multiplicity 1 eigenvalues, whereas we know $U(T)=d-5$. 
In the end, for multiplicity lists containing 2 as the largest multiplicity, any list containing $(2,2,2,2,2,2)$ is again ruled out by the fact that $U(T)=d-5$ by Theorem 4.8. Now, the elimination of impossible multiplicity lists is finished. Moreover, for all the lists remaining, there is an eigenvalue assignment.

In fact, we suspect a stronger statement than Theorem 4.12 is true.

Conjecture 4.13. For a diameter $d(\geqslant 7)$ core $T \in \mathcal{C}(d)$ such that no interior edges are subdivided, i.e., the ordered partition into four parts $(p, q, r, s)$ has $q=r=0$,

$$
\begin{aligned}
\mathcal{L}(T)= & \left\{4221^{d-3}, 421^{d-1}, 41^{d+1}, 32221^{d-4}, 3221^{d-2}, 321^{d}, 31^{d+2},\right. \\
& \left.222221^{d-5}, 22221^{d-3}, 2221^{d-1}, 2221^{d-1}, 221^{d+1}, 21^{d+3}, 1^{d+5}\right\} .
\end{aligned}
$$

Moreover, for $T \in \mathcal{C}(d)$ in which some interior edge is subdivided, i.e., the ordered partition into four parts $(p, q, r, s)$ has $q \neq 0$ or $r \neq 0$,

$$
\begin{aligned}
\mathcal{L}(T)= & \left\{4221^{d-3}, 421^{d-1}, 41^{d+1}, 3321^{d-3}, 331^{d-1}, 32221^{d-4}, 3221^{d-2}, 321^{d}, 31^{d+2},\right. \\
& \left.222221^{d-5}, 22221^{d-3}, 2221^{d-1}, 2221^{d-1}, 221^{d+1}, 21^{d+3}, 1^{d+5}\right\} .
\end{aligned}
$$

Acknowledgement: This work was in part supported by the National Science Foundation grant DMS \#0751964.

The authors thank our colleague, Professor Eric Swartz for suggesting an alternate and simple way to enumerate diameter $d$ cores as described in Proposition 3.9.

Data Availability Statement: Data sharing is not applicable to this article as no datasets were generated or analyzed during the current study.

\section{References}

[1] S.P. Buckley, J.G. Corliss, C.R. Johnson, C.A. Lombardía, and C.M. Saiago. Questions, conjectures, and data about multiplicity lists for trees. Linear Algebra Appl., 511 (2016), 72-109.

[2] W. Ding, M. Ingwersen, and C.R. Johnson. The minimum number of multiplicity 1 eigenvalues among real symmetric matrices whose graph is a 2-linear tree, Linear and Multilinear Algebra, submitted.

[3] W. Ding, and C.R. Johnson. The minimum number of multiplicity 1 eigenvalues among real symmetric matrices whose graph is a linear tree, in preparation.

[4] C.R. Johnson and A. Leal Duarte, The maximum multiplicity of an eigenvalue in a matrix whose graph is a tree, Linear and Multilinear Algebra, 46 (1999), 139-144.

[5] A. Leal Duarte and C.R. Johnson, On the minimum number of distinct eigenvalues for a symmetric matrix whose graph is a given tree, Mathematical Inequalities \& Applications, 5:2 (2002), 175-180.

[6] C.R. Johnson, A. Leal Duarte, and C. Saiago, Inverse eigenvalue problems and lists of multiplicities of eigenvalues for matrices whose graph is a tree: the case of generalized stars and double generalized stars, Linear Algebra Appl., 373 (2003), 311-330.

[7] C.R. Johnson, A. Leal Duarte, and C. Saiago, The Parter-Wiener theorem: refinement and generailzation, SIAM J. Matrix Anal. Appl., 25:2 (2003), 352-361.

[8] C.R. Johnson, A. Li, and A. Walker, Ordered multiplicity lists for eigenvalues of symmetric matrices whose graph is a linear tree, Discrete Mathematics, 333 (2014), 39-55.

[9] C.R. Johnson and C.M. Saiago, Branch duplication for the construction of multiple eigenvalues in an Hermitian matrix whose graph is a tree, Linear and Multilinear Algebra, 56:4 (2008), 357-380.

[10] C.R. Johnson and C. Saiago, Eigenvalues, Multiplicities and Graphs, Cambridge University Press, (2018).

[11] C.R. Johnson, B.D. Sutton, and A.J. Witt, Implicit construction of multiple eigenvalues for trees, Linear and Multilinear Algebra, 57 (2009), 409-420.

[12] C.R. Johnson, C. Jordan-Squire, and D. A. Sher, Eigenvalue assignments and the two largest multiplicities in a Hermitian matrix whose graph is a tree. Discrete Applied Mathematics, 158(6)(2010), 681-691.

[13] C.R. Johnson and T. Wakhare, The inverse eigenvalue problem for linear trees, Discrete Mathematics, 345(4)(2022), to appear.

[14] The On-Line Encyclopedia of Integer Sequences ${ }^{\circledR}$ (OEIS $®$ ), oeis.org/. 


\section{Appendix A Proof for Statements in Section 3.3}

Proof of Lemma 3.11. Consider what a diameter $d$ core with ordered partition $(p, q, r, s)$ looks like.

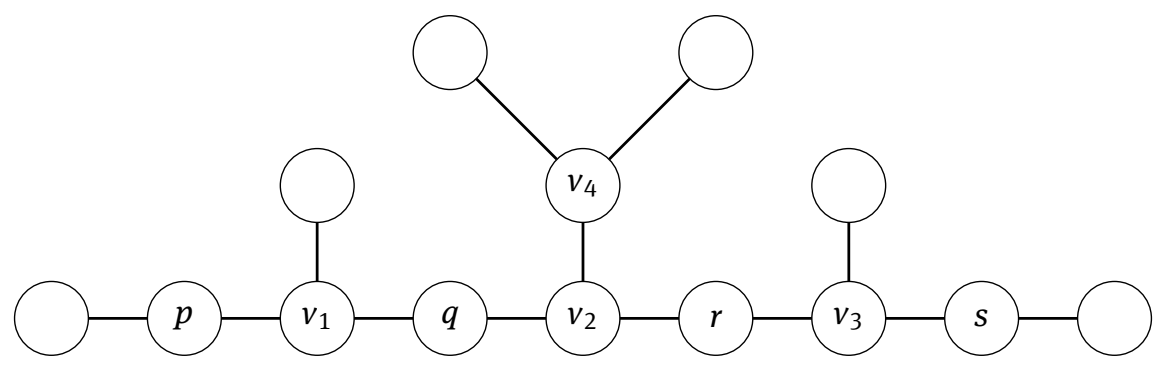

The key to diameters in a core is that they have to contain all the $p+q+r+s$ vertices resulting from edge subdivisions in the 10-vertex NL tree. First, whenever $p \neq 0$ and $s \neq 0$, the longest path in the core is uniquely determined, i.e., the horizontal path at the bottom in the graph. No other path is longer. Also, no matter what $q$ and $r$ are, possibly zero, this longest path contains them. Second, an ordered partition $\left(0,{ }^{\star}\right.$, ?, $\left.{ }^{\star}\right)$ requires either $p=0, q \neq 0$, and $s \neq 0$ or $s=0, r \neq 0$, and $p \neq 0$. Because these are symmetric, it suffices to consider the case when $p=0, q \neq 0$, and $s \neq 0$. Since $q \neq 0$, and $s \neq 0$, most part of diameters is determined; however, vertex $v_{1}$ has two pendents (because $p=0$ ), either of which can be part of a distinct diameter. Thus, such cores have two diameters. Third, cores with ordered partitions $\left({ }^{\star}, ?, 0,0\right)$ or $\left(0,{ }^{\star},{ }^{\star}, 0\right)$ have four diameters. On the one hand, ( $\left.{ }^{\star}, ?, 0,0\right)$ means $p \neq 0, r=0$, and $s=0$ (the symmetric case is omitted). All the diameters have to contain $v_{1}, p+1$ vertices on the left of $v_{1}, v_{2}$, and the $q$ vertices between $v_{1}$ and $v_{2}$ (possibly none). However, since $r=0$ and $s=0$, there are two distinct diameters containing $v_{4}$ and either of $v_{4}$ 's pendent vertices and another two distinct diameters containing $v_{3}$ and either of $v_{3}$ 's pendent vertices. Thus, such cores have a total of four distinct diameters. On the other hand, ordered partition $\left(0,{ }^{\star},{ }^{\star}, 0\right)$ means $p=s=0$, and $q, r \neq 0$. This implies that all the diameters contain $v_{1}, v_{2}, v_{3}$, and the $q$ and $r$ vertices in between. Then four pendent vertices - two of $v_{1}$ 's and two of $v_{3}$ 's - each result in a distinct diameter. Lastly, ordered partition $\left(0,{ }^{\star}, 0,0\right)$ means that $p=r=s=0$ and $q \neq 0$ (the symmetric case is omitted). Then all diameters have to contain $v_{1}, v_{2}$, and the $q$ vertices in between; in addition, one terminal vertex of the diameters needs to be one of the two pendent vertices of $v_{1}$, and the other terminal vertex needs to be one of $v_{3}$ 's and $v_{4}$ 's four pendent vertices. Thus, cores with such ordered partition have eight distinct diameters.

Proof of Corollary 3.12. Up to forward/backward symmetry, ordered partitions discussed in Lemma 3.11, namely, $\left({ }^{\star}, ?, ?, \star\right),\left(0, \star, ?,{ }^{\star}\right),(\star, ?, 0,0),\left(0, \star{ }^{\star}, 0\right)$, and $(0, \star, 0,0)$, cover all the possibilities. In particular, $\left({ }^{\star}, ?, 0,0\right)$ and $\left(0,{ }^{\star}, 0,0\right)$ account for ordered partitions with one nonzero part, $\left(\star^{\star}, ?, ?,{ }^{\star}\right),\left(0,{ }^{\star}, ?,{ }^{\star}\right)$, $\left({ }^{\star}, ?, 0,0\right)$, and $\left(0,{ }^{\star}, \star, 0\right)$ account for ordered partitions with two nonzero parts, $\left(0,{ }^{\star}, ?,{ }^{\star}\right)$ and $\left(\star, ?, ?,{ }^{\star}\right)$ account for ordered partitions with three nonzero parts, and $\left({ }^{\star}, ?, ?,{ }^{\star}\right)$ accounts for ordered partitions with four nonzero parts. Therefore, a diameter $d$ core $(d \geqslant 7)$ could only possibly have 1, 2, 4 or 8 distinct diameters. Note that $d \geqslant 6$ guarantees that $(p, q, r, s)$ is a partition of a positive integer; however, for the only diameter 5 core, i.e., with ordered partition $(0,0,0,0)$, have twelve distinct diameters.

Furthermore, for $\mathrm{C}(d)$ such that $d \geqslant 7$, each of $\{1,2,4,8\}$ will occur as the number of distinct diameters for at least one of the cores. In particular, when $d \geqslant 7, p+q+r+s \geqslant 2$; also, none of the ordered partitions in form of $\left(\star, ?, ?,{ }^{\star}\right),\left(0,{ }^{\star}, ?,{ }^{\star}\right),\left({ }^{\star}, ?, 0,0\right),\left(0,{ }^{\star},{ }^{\star}, 0\right)$, and $\left(0,{ }^{\star}, 0,0\right)$ requires more than two nonzero parts. Thus, each of $\{1,2,4,8\}$ will occur for at least one of the cores.

Proof of Corollary 3.13. First, the number of cores with a unique diameter is a doubly lagged function of $|\mathcal{C}(d)|$. By Lemma 3.11, such cores have ordered partitions in the form of $\left({ }^{\star}\right.$, ?, ? , *). In other words, any diameter $d$ core with $p \geqslant 1$ and $s \geqslant 1$ has a unique diameter. Recall Algorithm 3.6, compared to generating all $|\mathcal{C}(d)|$ diameter $d$ cores from $d-5$ edge subdivisions on four edges in one diameter, we now mandate two edge subdivisions to make $p=1$ and $s=1$. Then the rest $d-7$ edge subdivisions can be applied on any of the four edges, which generates $|\mathcal{C}(d-2)|$ cores with a unique diameter. 
Second, cores with two diameters have ordered partitions in the form of $\left(0,{ }^{\star}, ?,{ }^{\star}\right)$. The 0 restricts us to apply $d-5$ edge subdivisions on only three edges; in addition, two *'s requires two edge subdivisions on respective edges and leaves us with $d-7$ edge subdivisions on three edges. This boils down to the number of ways that $d-7$ identical balls can be partitioned into three groups (empty groups are allowed), which is equivalent to inserting two walls into $(d-7)+1$ spaces among a line of $d-7$ objects. Thus, there are $\left(\begin{array}{c}d-6 \\ 2\end{array}\right)$ such partitions. Moreover, isomorphism due to forward/backward symmetry does not arise because of the 0 in the ordered partition. Thus, $\left(\begin{array}{c}d-6 \\ 2\end{array}\right)$ diameter $d$ cores have two diameters.

Third, the number of cores with four diameters is the sum of the number of cores with ordered partitions in the form of $\left({ }^{\star}, ?, 0,0\right)$ or $\left(0,{ }^{\star},{ }^{\star}, 0\right)$. On the one hand, ordered partition $\left({ }^{\star}, ?, 0,0\right)$ is similar to $\left(0,{ }^{\star}\right.$, ? , *). So, one edge subdivision is mandated by the ${ }^{\star}$, and the rest $d-6$ edge subdivisions need to be applied to two edges. Thus, there are $\left(\begin{array}{c}d-6+1 \\ 1\end{array}\right)=d-5$ cores with ordered partitions $(\star$, ?, 0,0$)$. Again, there is no forward/backward symmetry issue for $(\star, ?, 0,0)$. On the other hand, cores with ordered partitions $(0, \star, \star, 0)$ have two mandated edge subdivisions by the two *'s and $d-7$ edge subdivisions left, so there are $\left(\begin{array}{c}d-7+1 \\ 1\end{array}\right)=d-6$ ways to partition $d-7$ edge subdivisions to two edges. However, we now encounter the double counting problem due to forward/backward symmetry for order partitions $\left(0,{ }^{\star},{ }^{\star}, 0\right)$. Again, just as Proposition 3.9, parity of $d$ makes a difference. When $d$ is even, $d-7$ is odd, every core is counted exactly twice by the symmetry, so there are $(d-6) / 2$ cores with ordered partitions $\left(0,{ }^{\star},{ }^{\star}, 0\right)$; when $d$ is odd, $d-7$ is even, every core is counted exactly twice by the symmetry except the core with ordered partition $(p, q, r, s)$ such that $p=s=0$ and $q=r$, so there are $(d-6) / 2+1 / 2$ cores with ordered partitions $\left(0,{ }^{\star},{ }^{\star}, 0\right)$. To summarize, $\lceil(d-6) / 2\rceil+(d-5)$ diameter $d$ cores have four diameters.

Lastly, the number of cores with eight diameters in $\mathcal{C}(d)$ is 1 because the ordered partition $\left(0,{ }^{\star}, 0,0\right)$ is unique. 